\title{
Coronectomia intencional como alternativa para evitar lesões ao nervo alveolar inferior: Relato de caso clínico
}

\author{
Intentional coronectomy as an alternative to avoid injuries to the lower alveolar nerve: Clinical case \\ report
}

Coronectomía intencional como alternativa para evitar lesiones del nervio alveolar inferior: Reporte de caso clínico

Recebido: 14/07/2021 | Revisado: 19/07/2021 | Aceito: 22/07/2021 | Publicado: 31/07/2021

\author{
Maria Emanuella Letícia da Silva \\ ORCID: https://orcid.org/0000-0002-7159-1165 \\ Centro Universitário Brasileiro, Brasil \\ E-mail: emanuelaleticia@hotmail.com \\ Izabella Maria Barbosa da Silva \\ ORCID: https://orcid.org/0000-0002-4249-4681 \\ Universidade Federal de Pernambuco, Brasil \\ E-mail: izabella.ufpe@hotmail.com \\ Renata de Albuquerque Cavalcanti Almeida \\ ORCID: https://orcid.org/0000-0003-1101-3491 \\ Universidade de Pernambuco, Brasil \\ E-mail: realmeida81@outlook.com
}

\begin{abstract}
Resumo
Introdução: O dano ao Nervo Alveolar Inferior (NAI) é fator de risco significativo após remoção de terceiro molar impactado. São tidos como sinais considerados de alto risco de lesão nervosa a ausência de osso cortical entre a raiz dental e o canal mandibular, bem como a constrição do mesmo por onde passa a raiz do dente. Objetivo: Relatar um caso de coronectomia intencional de terceiro molar inferior direito, devido contato íntimo com o canal mandibular. Paciente, sexo feminino, 19 anos, compareceu ao Centro de Especialidades Odontológicas do Recife, indicada para exodontia dos terceiros molares. A radiografia panorâmica revelou impactação horizontal do dente 48, contato da coroa com as raízes do segundo molar e aparente contato com o NAI. Solicitou-se tomografia computadorizada de feixe cônico, confirmando íntima relação. A conduta escolhida foi realização de coronectomia intencional e sepultamento das raízes. Conclusão: A literatura comprova que essa técnica reduz significativamente a lesão do nervo alveolar inferior sendo uma alternativa eficaz e segura para evitar complicações nervosas.
\end{abstract}

Palavras-chave: Dente impactado; Terceiro molar; Cirurgia bucal.

\begin{abstract}
Introduction: Damage to the Inferior Alveolar Nerve (NAI) is a significant risk factor after removal of an impacted third molar. The absence of cortical bone between the dental root and the mandibular canal, as well as it's constriction through which the tooth's root passes, are considered signs of high risk of nerve damage. Objective: To report a case of intentional coronaectomy of the lower right third molar, due to intimate contact with the mandibular canal. Patient, female, 19 years old, attended the Dental Specialties Center in Recife, indicated for third molar extraction. Panoramic radiography revealed horizontal impaction of tooth 48 , crown contact with second molar roots, and apparent contact with the NAI. Cone beam computed tomography was requested, confirming a close relationship. The chosen approach was intentional coronaectomy and root burial. Conclusion: Literature proves that this technique significantly reduces injury to the inferior alveolar nerve, being an effective and safe alternative to avoid nerve complications.
\end{abstract}

Keywords: Impacted tooth; Third molar; Oral surgery.

\section{Resumen}

Introducción: El daño del nervio alveolar inferior (NAI) es un factor de riesgo significativo después de la extracción de un tercer molar impactado. La ausencia de hueso cortical entre la raíz dental y el canal mandibular, así como su constricción por donde pasa la raíz del diente, se consideran signos de alto riesgo de daño nervioso. Objetivo: Reportar un caso de coronaectomía intencional del tercer molar inferior derecho, por contacto íntimo con el canal mandibular. Paciente, mujer de 19 años, acudió al Centro de Especialidades Dentales de Recife, indicada para extracción de tercer molar. La radiografía panorámica reveló impactación horizontal del diente 48, contacto de la corona con las raíces del segundo molar y contacto aparente con el NAI. Se solicitó tomografía computarizada de haz cónico, confirmando una estrecha relación. El abordaje elegido fue la coronaectomía intencional y el entierro radicular. Conclusión: La literatura 
demuestra que esta técnica reduce significativamente la lesión del nervio alveolar inferior, siendo una alternativa eficaz y segura para evitar complicaciones nerviosas.

Palabras clave: Diente impactado; Tercer molar; Cirugía oral.

\section{Introdução}

A remoção cirúrgica de terceiro molar inferior impactado é um dos procedimentos mais frequentemente realizados pelo Cirurgião Bucomaxilofacial, seja para fins terapêuticos ou profiláticos, sendo frequentemente associados a dor, edema e trismo pós-operatório (Mukherjee; Vikraman; Duraiswamy Sankar, 2016).

Uma possível complicação em decorrência de exodontias dos terceiros molares inferiores é a lesão do nervo alveolar inferior, tendo em vista a íntima relação entre eles, resultando em parestesia, hipoestesia ou disestesia do lábio inferior, dentes, gengiva e pele sobre o queixo, o que afeta significativamente a qualidade de vida do paciente (Mukherjee; Vikraman; Duraiswamy Sankar, 2016; Crameri; Kuttenberger, 2018).

A lesão nervosa causa uma sensação alterada temporária ou permanente para as regiões onde esses nervos sensoriais inervam. Sensação de perda de sensibilidade, formigamento, sensação anormal ou sensação dolorosa podem ser observadas. O risco de lesão temporária é relatado em $5 \%$ dos casos enquanto os danos permanentes são relatados em $0,2 \%$ dos casos. A literatura relata que as lesões nervosas melhoram significativamente em 3 a 6 meses, estimando-se que 96\% dos distúrbios neurossensoriais se recuperam dentro de 4-8 semanas (Barraclough; Power; Pattni, 2017; Hasegawa et al., 2018).

Segundo Yeung et al. (2018) e Tachinami et al. (2017), a parestesia tem sido associada a fatores como idade, sexo, tipo de anestesia e a experiência do cirurgião. No entanto, vários estudos relataram a importância da avaliação tomográfica para prevenção de lesões nervosas.

Com a crescente solicitação de tomografia computadorizada de feixe cônico (TCFC) como exame complementar de imagem para pacientes com indicação de exodontia de dentes inclusos, o relacionamento do nervo aveolar inferior com dentes inclusos mandibulares, pode agora ser apurado com precisão a partir de suas raízes. Os sinais tridimensionais de imagem dessa relação são visualizados nos cortes coronais. São tidos como sinais considerados de alto risco de lesão nervosa a ausência de osso cortical entre a raiz dental e o canal nervoso, bem como a constrição do canal mandibular por onde passa a raiz do dente. Essas imagens auxiliam o cirurgião a indicar, contraindicar ou modificar sua técnica para a realização dos procedimentos cirúrgicos (Bhola; Pellatt, 2018; Crameri; Kuttenberger, 2018).

Diante de terceiros molares inferiores, podem ser indicados como tratamento a exodontia, a proservação ou ainda, a coronectomia. A coronectomia, também conhecida como remoção parcial da raiz ou odontectomia parcial, é mais comumente considerada para os dentes terceiros molares inferiores, nos quais as raízes estão intimamente associadas ao nervo alveolar inferior (NAI), para reduzir o risco de danos ao nervo (Espert et al., 2016; Ali; Benton; Yates, 2018;).

Existem algumas controvérsias sobre o procedimento de coronectomia quando comparada a exodontia do dente como um todo. Essas controvérsias estão relacionadas à falta de conhecimento a longo prazo dos remanescentes radiculares e possíveis complicações como dor pós-operatória, cavidade seca ou migração radicular após algum tempo da cirurgia (Biočanin; Todorović, 2014; Cosola et al., 2020).

Infecções e a necessidade de segundo procedimento para remoção de raiz após sua migração são as complicações mais frequentes associadas a realização de coronectomias. A Migração pós-operatória das raízes é considerada uma complicação a longo prazo e é um achado comum. Quando de sua ocorrência, termina por afastar as raízes do Nervo Alveolar Inferior, facilitando o segundo procedimento e diminuindo o risco de lesão nervosa. Pedersen et al. (2018) e Pitros et al. (2019) alegam que essa migração pode ocorrer até 10 anos após procedimento. 
O objetivo deste estudo foi relatar um caso clínico de coronectomia de terceiro molar inferior incluso cujas raízes apresentavam-se em íntima relação com o canal mandibular, visando diminuir o dano ao nervo alveolar inferior e suas consequências.

\section{Relato de Caso}

Paciente KFS, feoderma, sexo feminino, 19 anos, compareceu ao Centro de Especialidades Odontológicas da cidade do Recife, com indicação de exodontia dos terceiros molares. A paciente não apresentava nenhuma doença sistêmica. Foi solicitada radiografia panorâmica (Figura 1A) e constatou-se que o terceiro molar inferior direito (48) estava incluso horizontalmente, contatando as raízes do segundo molar inferior e com aparente contato com o nervo alveolar inferior (NAI).

Foi solicitada uma tomografia computadorizada de feixe cônico da mandíbula, confirmando-se intima relação do dente com o NAI (Figura 1B).

Figura 1A: Radiografia panorâmica inicial; B: Tomografia computadorizada de feixe cônico confirmando íntima relação do dento com o NAI.

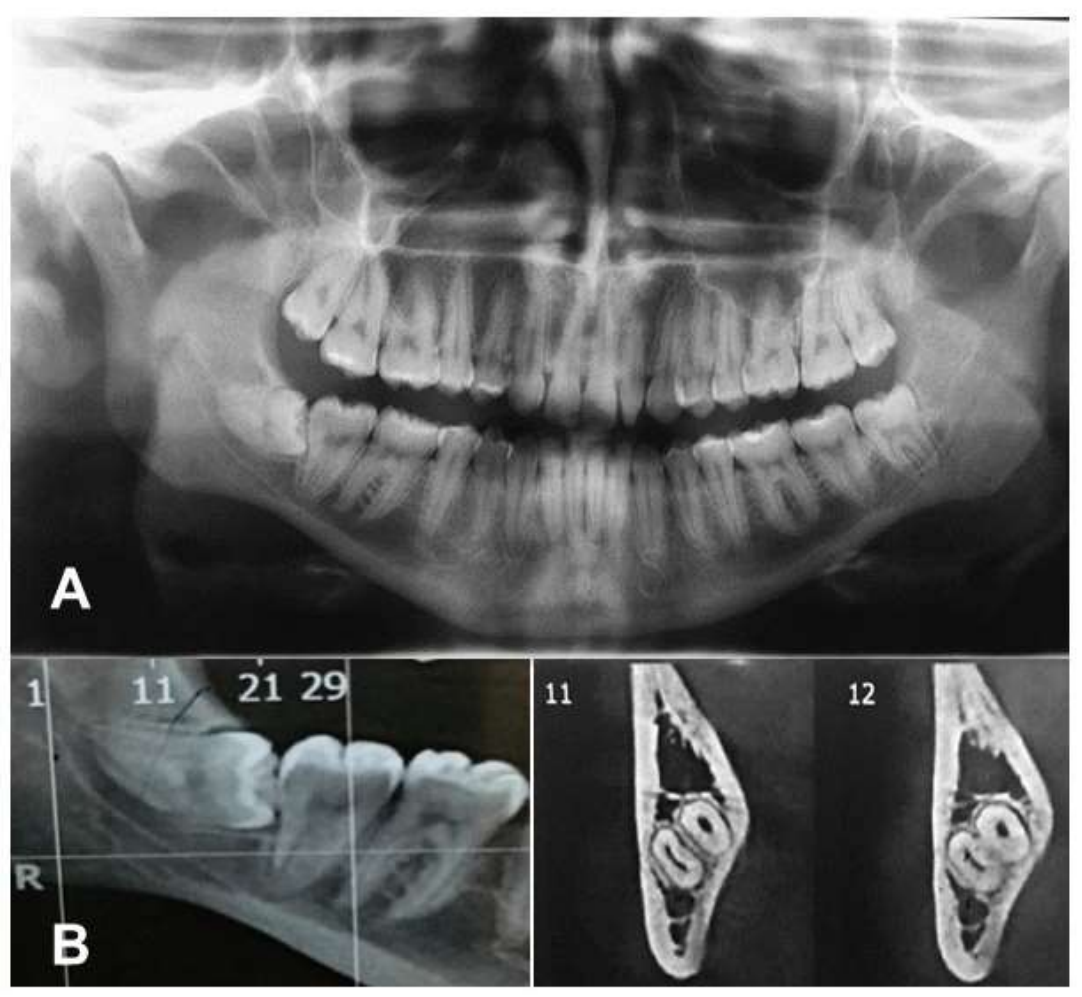

Fonte: Autores.

Visando a prevenção da reabsorção radicular do dente 47 em decorrência do dente 48 incluso, duas eram as possibilidades terapêuticas: exodontia ou coronectomia. A paciente foi informada dos riscos associados à exodontia do dente 48 e aos benefícios e às possíveis complicações, concordando com a realização da coronectomia intencional e consequente sepultamento das raízes, tendo em vista a existência de íntima relação com o NAI.

O procedimento cirúrgico foi realizado sob anestesia local, com bloqueio do nervo alveolar inferior, lingual e bucal, utilizando-se como anestésico local o cloridrato de mepivacaína a 2\% com adrenalina 1:100.000 (Nova DFL, São Paulo, Brasil). Dexametasona foi utilizada de forma preemptiva na dose de $8 \mathrm{mg}$, uma hora antes do procedimento. Um retalho do tipo envelope foi confeccionado e rebatido (Figura 2A), iniciando-se, pois, a osteotomia da tábua óssea vestibular com auxílio da caneta de 
alta rotação e broca cirúrgica no 702 (JET, São Paulo, Brasil) sob irrigação constante com soro fisiológico, com objetivo de expor toda a coroa (Figura 2B). Prosseguiu-se com a odontosecção (Figura 2C), separando a coroa da raíz a nível da união amelocementária, removendo-se a porção coronária e sepultando as raízes (figura 2D).

Figura 2A: Confecção do retalho envelope; B: Osteotomia para exposição da coroa do dente; C: Odontosecção para remoção da porção coronária; D: Coroa removida.

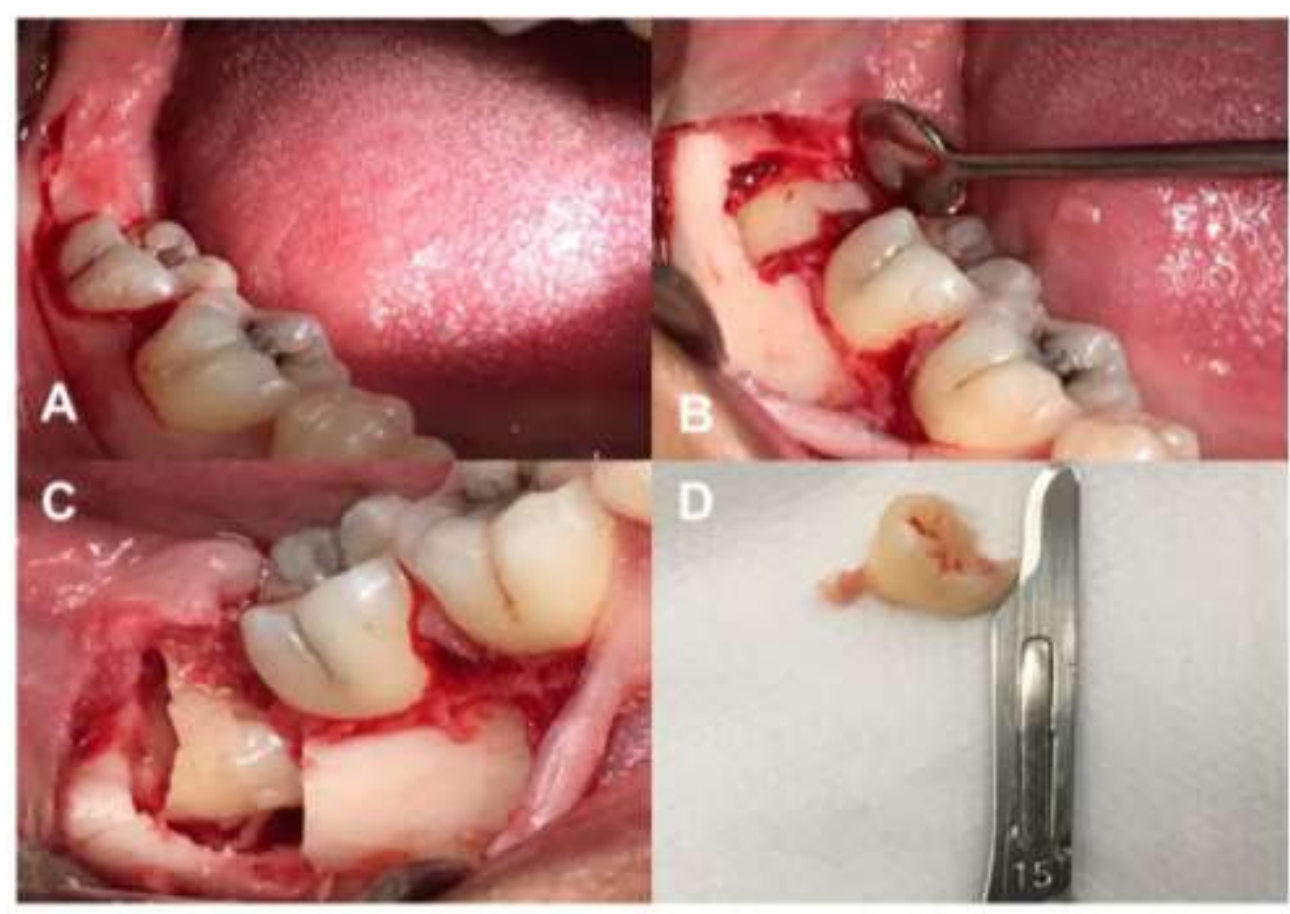

Fonte: Autores.

Em seguida, fez-se a regularização do remanescente radicular, lavagem abundante da ferida com soro fisiológico e sutura com fio de seda 3-0 (Ethicon, São Paulo, Brasil) por primeira intenção (Figura 3A).

Foram prescritas como medicações pós-operatórias amoxicilina de $500 \mathrm{mg}$, a cada oito horas por um período de sete dias, além de ibuprofeno de $600 \mathrm{mg}$ e dipirona de $500 \mathrm{mg}$, a cada seis horas, por um período de três e dois dias respectivamente. A paciente evolui bem e teve sua sutura removida após sete dias da cirurgia. Solicitou-se radiografia panorâmica após 01 ano do procedimento para acompanhamento do caso e ver se houve alguma alteração em relação as raízes (Figura 3B). 
Figura 3A: Sutura com fio de seda 3-0; B: Radiografia panorâmica após 01 ano para acompanhamento.

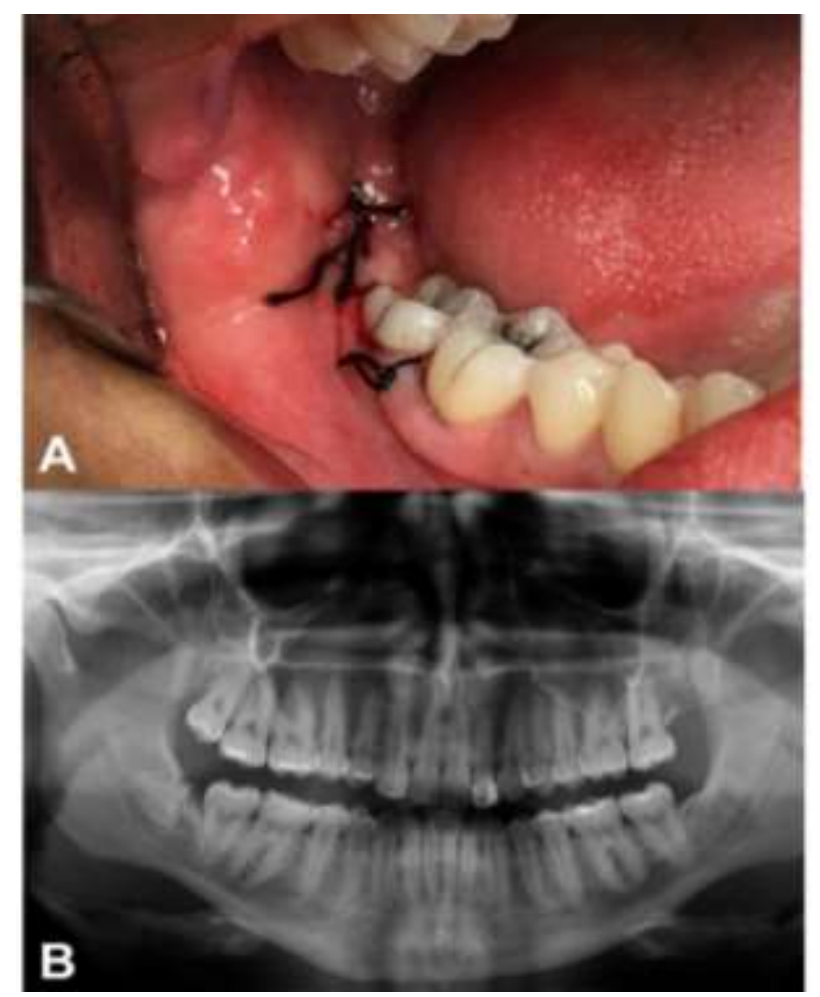

Fonte: Autores.

\section{Discussão}

De acordo com os estudos de Hasegawa et al. (2018) e Tachinami et al. (2017), a exodontia de terceiros molares foi a causa do dano nervoso em 52,1\% dos casos de cirurgias orais. As lesões do nervo alveolar inferior (NAI) geralmente ocorrem como complicação de uma exodontia do terceiro molar inferior, variando de $0,5 \%$ a $8 \%$. Diante dessa estimativa, é prudente, diante de exodontias de terceiros molares inferiores, reconhecer a relação de proximidade desse com o canal alveolar, tendo em vista a possibilidade de prevenção.

De acordo com Fee et al. (2016) e Luo et al. (2018), a radiografia panorâmica (RP) é a ferramenta de diagnóstico padrão para esse fim, no entanto, se a avaliação da radiografia panorâmica indica uma relação íntima entre o terceiro molar e o canal mandibular, uma investigação adicional usando a tomografia computadorizada de feixe cônico (TCFC) deve ser recomendada para verificar a relação em três dimensões. Baseado nas evidências disponíveis, no caso em questão, solicitou-se TCFC devido à proximidade das raízes com o canal mandibular observada na RP, onde confirmou-se ausência de cortical entre as raízes e o canal mandibular.

Uma revisão sistemática realizada por Barraclough et al. (2017) comparou as técnicas de coronectomia em relação a remoção cirúrgica completa de terceiros molares, afim de contrastar qual das duas técnicas oferecia mais risco de lesão ao nervo aveolar inferior. Foi observado que a lesão temporária do nervo aveolar inferior ocorreu de forma mais frequente quando da remoção completa do dente em até $19 \%$ dos casos em comparação às coronectomias (0-9,5\% dos casos).

O trabalho de Pedersen et al. (2018) avaliou 231 casos de 191 pacientes que foram submetidos à coronectomia e acompanhados por um período de 5,7 anos. Foi observado como complicação associada a essa técnica, a migração das raízes e a necessidade de um segundo procedimento cirúrgico. No entanto, essa migração termina facilitando o segundo procedimento e diminui o risco de lesão nervosa associada ao procedimento. A possibilidade da realização de coronectomia como tratamento para o paciente, ao invés da exodontia, e as possíveis complicações associadas a cada caso devem ser passadas sempre aos 
pacientes para que esses participem dessa escolha e consintam o tratamento. A paciente do caso em questão estava ciente dos riscos e benefícios associados, onde o procedimento escolhido foi a coronectomia, diante da possibilidade de lesão nervosa.

Uma revisão sistemática de Barcellos et al. (2019) traz como objetivo identificar fatores associados a reincidência de um novo procedimento após coronectomia do terceiro molar inferior. Fizeram parte da amostra 1.664 pacientes e 2.062 dentes foram submetidos à coronectomia. A necessidade de reoperar o paciente submetido a tal procedimento foi considerado baixa. Apesar da reoperação do paciente ser considerada uma desvantagem técnica, não representa falha, e ainda é uma alternativa segura para o tratamento de terceiros molares intimamente ligados ao canal mandibular.

Diante de lesões nervosas após exodontias, uma conduta bastante realizada é o tratamento medicamentoso, onde o uso de vitaminas, especialmente as do complexo B, anti-inflamatórios e antineuríticos, é amplamente utilizada para o tratamento da parestesia, diminuindo o tempo de duração do quadro (Lopes; Freitas, 2013; Ribeiro et al., 2021).

O Fosfato Dissódico de citidina (CMP) e o Trifosfato Trissódico de uridina (UTP) são exemplos de co-enzimas consideradas essenciais para a regeneração nervosa. Uma medicação conhecida como ETNA apresenta essas duas coenzimas associadas ao Acetato de hidroxicobalamina, fornecendo ao organismo esses nucleotídeos e Vitamina B12 favorecendo a reparação da lesão nervosa e a recuperação da sensibilidade, sua prescrição é uma excelente conduta quando do diagnóstico de lesões nervosas após os procedimentos cirúrgicos (Oliveira, 2018).

\section{Conclusão}

Apesar da controvérsia sobre a efetividade do procedimento da coronectomia como alternativa de tratamento para os terceiros molares inferiores intimamente associados ao canal mandibular, a literatura comprova que essa técnica reduz significativamente a lesão do nervo alveolar inferior, mesmo em casos de alto risco de lesão, sendo uma alternativa eficaz e segura para evitar complicações nervosas e que pode ser muito bem indicada para os pacientes.

\section{Referências}

Ali, A. S., Benton, J. A., \& Yates, J. M. (2018). Risk of inferior alveolar nerve injury with coronectomy vs surgical extraction of mandibular third molars-A comparison of two techniques and review of the literature. Journal of oral rehabilitation, 45(3), 250-257.

Barcellos, B. M., Velasques, B. D., Moura, L. B., \& Xavier, C. B. (2019). What are the parameters for reoperation in mandibular third molars submitted to coronectomy? A systematic review. Journal of Oral and Maxillofacial Surgery, 77(6), 1108-1115.

Barraclough, J., Power, A., \& Pattni, A. (2017). Treatment planning for mandibular third molars. Dental update, 44(3), 221-228.

Bhola, S., \& Pellatt, A. (2018). Coronectomies: assessment and treatment planning. British dental journal, 225(2), $125-12$.

Biočanin, V., \& Todorović, L. (2014). Coronectomy of two neighbouring ankylosed mandibular teeth: A case report. Vojnosanitetski pregled, 71(8), 777-779.

Cosola, S., Kim, Y. S., Park, Y. M., Giammarinaro, E., \& Covani, U. (2020). Coronectomy of Mandibular Third Molar: Four Years of Follow-Up of 130 Cases. Medicina, 56(12), 654 .

Crameri, M., \& Kuttenberger, J. J. (2018). Application and evaluation of coronectomy in Switzerland. Swiss Dent J, 128(7-8), 582-586.

Espert, J. C., Martínez, S. P., Ballester, J. C., Oltra, D. P., \& Diago, M. P. (2016). Coronectomy of impacted mandibular third molars: a meta-analysis and systematic review of the literature. Medicina oral, patología oral y cirugía bucal. Ed. inglesa, 21(4), 16.

Falci, S. G. M., Lima, T. C., Martins, C. C., Santos, C. R. R. D., \& Pinheiro, M. L. P. (2017). Preemptive effect of dexamethasone in third-molar surgery: a meta-analysis. Anesthesia progress, 64(3), 136-143.

Fee, P. A., Wright, A., \& Cunningham, C. (2016). Cone beam computed tomography in pre-surgical assessment of mandibular third molars. Evidence-based dentistry, 17(4), 117-118.

Hasegawa, T., Yamada, S. I., Ueda, N., Soutome, S., Funahara, M., Akashi, M., ... \& Komori, T. (2018). Treatment modalities and risk factors associated with refractory neurosensory disturbances of the inferior alveolar nerve following oral surgery: a multicentre retrospective study. International journal of oral and maxillofacial surgery, 47(6), 794-801.

Lopes, G. B., \& Freitas, J. B. (2013). Parestesia do nervo alveolar inferior após exodontia de terceiros molares. Arquivo Brasileiro de Odontologia, 9(2), 35-40. 
Research, Society and Development, v. 10, n. 9, e46710918270, 2021 (CC BY 4.0) | ISSN 2525-3409 | DOI: http://dx.doi.org/10.33448/rsd-v10i9.18270

Luo, Q., Diao, W., Luo, L., \& Zhang, Y. (2018). Comparisons of the computed tomographic scan and panoramic radiography before mandibular third molar extraction surgery. Medical science monitor: international medical journal of experimental and clinical research, $24,3340$.

Mukherjee, S., Vikraman, B., \& Duraiswamy Sankar, M. S. V. (2016). Evaluation of outcome following coronectomy for the management of mandibular third molars in close proximity to inferior alveolar nerve. Journal of clinical and diagnostic research: JCDR, 10(8), ZC57.

Oliveira, K. D. C. M. D. (2018). Eficácia da laserterapia e da laseracupuntura no tratamento de parestesia em pacientes submetidos à cirurgias de implantes e extração de terceiros molares inferiores (Doctoral dissertation, Universidade de São Paulo).

Pedersen, M. H., Bak, J., Matzen, L. H., Hartlev, J., Bindslev, J., Schou, S., \& Nørholt, S. E. (2018). Coronectomy of mandibular third molars: a clinical and radiological study of 231 cases with a mean follow-up period of 5.7 years. International journal of oral and maxillofacial surgery, $47(12), 1596-1603$.

Pitros, P., Jackson, I., \& O’Connor, N. (2019). Coronectomy: a retrospective outcome study. Oral and maxillofacial surgery, 23(4), 453-458.

Ribeiro, A. C. F., dos Anjos Gonçalves, T., Carneiro, M. N., \& Carneiro, P. M. A. (2021). O uso da laserterapia associada ao complexo B na prevenção de parestesia do nervo mandibular pós ressecção cirúrgica de ceratocisto odontogênico: relato de caso. Revista Eletrônica Acervo Saúde, 13 (2), e5626-e5626.

Tachinami, H., Tomihara, K., Fujiwara, K., Nakamori, K., \& Noguchi, M. (2017). Combined preoperative measurement of three inferior alveolar canal factors using computed tomography predicts the risk of inferior alveolar nerve injury during lower third molar extraction. International journal of oral and maxillofacial surgery, 46(11), 1479-1483.

Yeung, A. W. K., Wong, N. S. M., Bornstein, M. M., \& Leung, Y. Y. (2018). Three-dimensional radiographic evaluation of root migration patterns 4-8.5 years after lower third molar coronectomy: a cone beam computed tomography study. International journal of oral and maxillofacial surgery, 47(9), $1145-1152$. 